In Crescendo, 2017; 8(2): 267-280

Fecha de recepción: 31 de mayo del 2017

Fecha de aceptación: 3 de noviembre del 2017

\title{
PARTICIPACIÓN DE LA COMUNA 21 DE CALI EN EL ORDENAMIENTO TERRITORIAL
}

\author{
PARTICIPATION OF THE COMMUNE 21 OF CALI \\ IN THE TERRITORIAL ORDER
}

\author{
Wilson Romero Palacios ${ }^{1}$, Ana Mercedes Ocampo Hoyos ${ }^{2}$, \\ José Fabián Ríos Obando ${ }^{3}$
}

\section{RESUMEN}

El proyecto tiene como principal objetivo, analizar el Plan de Ordenamiento Territorial (POT) desde la dimensión social de la sostenibilidad. Para tal fin, se describirá la participación de la sociedad civil de la comuna 21 de Cali. La presente investigación parte de una necesidad sentida en la ciudad de Cali, en donde se observa de manera sistemática una desarticulación, entre la política pública en relación a la capacidad de respuesta a las carencias que presenta la población, en cuanto a la atención de consideraciones sociales y ambientales, e incluso económicas, de la población vulnerable para garantizar el desarrollo sostenible para un crecimiento armónico de la ciudad. Para esta finalidad se entrevistó a una muestra de 400 personas en la comuna 21, con algún grado de escolaridad distribuidas de la siguiente manera: 204 con Bachillerato, 72 con nivel Técnico, 68 con nivel Tecnológico, 56 Universitarios. Se empleó una encuesta. Se encontró que en la gran mayoría 74\% (148 encuestados) no conocen el sentido del POT, el 82,5\% (165 encuestados) no reconocen el POT de la ciudad de Cali y el 100\%

1 Vicerrector Académico, Corporación Universitaria Centro Superior Unicuces, Candidato a Doctor en Administración de Negocios, Grupo de Investigación Construcecs, viceacademica@unicuces.edu.co

2 Directora Investigación Corporación Universitaria Centro Superior. Doctora Geografía e Historia Planificación Territorialy Gestión Ambiental, investigación@unicuces.edu.co

3 Estudiante de Doctorado en Administración. Maestría en Medio Ambiente y Desarrollo Sostenible. Corporación Universitaria Centro Superior. Líder Grupo de Investigación Unirse. 
nunca tuvo participación para su construcción. De igual manera, el 89,5 (179 encuestados) no conocen las implicaciones para la comuna donde habitan y el 87,5\% (175 encuestados) no consideran o no saben que el POT puede afectar su calidad de vida. La investigación evidencia la falta de promoción de espacios, por un lado y de participación ciudadana por el otro, para que adquiera sentido el desarrollo urbano sostenible.

Palabras ClaVe: Desarrollo, Ordenamiento, Participación Social, Sostenibilidad, Territorialidad.

\section{ABSTRACT}

The main objective of the project is to analyze the Territorial Ordering Plan (POT) from the social dimension of sustainability. For this purpose, the participation of the civil society of Commune 21 of Cali will be described. The present investigation is based on a felt need in the city of Cali, where a disarticulation is observed in a systematic way, between the public policy in relation to the capacity of response to the deficiencies that the population presents, as regards the attention of considerations social and environmental, and even economic, of the vulnerable population to guarantee sustainable development for a harmonious growth of the city. For this purpose, a sample of 400 people was interviewed in commune 21, with some degree of schooling distributed as follows: 204 with Baccalaureate, 72 with Technical level, 68 with Technological level, 56 University students. A survey was used. It was found that in the great majority 74\% (148 respondents) do not know the meaning of the POT, $82.5 \%$ (165 respondents) do not recognize the POT of the city of Cali and 100\% never had participation for its construction. Similarly, 89.5 (179 respondents) do not know the implications for the commune where they live and $87.5 \%$ (175 respondents) do not consider or do not know that the POT can affect their quality of life. The research shows the lack of promotion of spaces, on the one hand and citizen participation on the other, so that sustainable urban development acquires meaning.

KEY WORDS: Development, Social Participation, Planning, Sustainability, Territoriality.

\section{INTRODUCCIÓN}

El proceso de descentralización adelantado en Colombia con ocasión a la Constitución Política de Colombia envistió de poder y reconocimiento a los municipios para orientar de manera autónoma y participativa el ordenamiento territorial, en la que de acuerdo a la Constitución Política de Colombia, se delegaba a cada Municipio el deber de "prestar los servicios públicos que determine la ley, construir las obras que demande el progreso local, ordenar el desarrollo de su territorio, promover la participación comunitaria, el mejoramiento social y cultural de sus habitantes y cumplir las demás funciones que le asignen la Constitución y las leyes" República de Colombia (1991). 
El objetivo de dicha reestructuración está dirigido a disminuir las disparidades y brechas de desarrollo entre Municipios, sentando las bases para consolidar un desarrollo simétrico a nivel nacional, toda vez que de acuerdo con Devia (2008) "el principal objetivo del municipio es servir a su comunidad, por medio de la generación de los servicios sociales, garantizando la satisfacción de las necesidades mínimas y básicas, de manera eficiente y eficaz, en particular los servicios domiciliarios y las condiciones mínimas habitacionales".

El POT, como instrumento técnico y normativo de planeación a largo plazo, concebido mediante la Ley 388 de 1997 y demás decretos reglamentarios, propende por orientar el desarrollo territorial de los municipios, mediante estrategias y otros instrumentos que propenda por armonizar dichos intereses con las consideraciones sociales, analizadas desde la sostenibilidad.

Por lo anterior, se requiere de una articulación entre las demás actuaciones político administrativas de cara a consolidar acciones vinculantes que posibiliten el alcance de los componentes y objetivos definidos dentro de la visión establecida por la herramienta en mención.

El Plan de Ordenamiento es entonces "el instrumento básico del ordenamiento territorial. Ocupa en el ámbito municipal, el primer lugar jerárquico en la relación de instrumentos de planeamiento. En efecto, todos los instrumentos que posteriormente lo desarrollen, deben ajustarse a las previsiones contenidas en él" Departamento Administrativo de Planeación Municipal (1999).

Resulta interesante entonces analizar los POT desde un componente social, toda vez que la política pública debe crear escenarios que respondan a las necesidades colectivas en armonía con la conservación de los bienes y servicios ambientales, siendo prioritario adelantar investigaciones que permitan resignificar la participación de la sociedad civil, en palabras de Rodríguez y Reyes (2008) “emerge la importancia de desarrollar metodologías e instrumentos de ordenamiento territorial con un enfoque estratégico vinculante, cuya imagen objetivo de largo plazo sea la sustentabilidad".

Mediante el Acuerdo No 0373 de 2014, el Concejo Municipal de Cali, dio lugar a la revisión ordinaria del contenido de largo plazo del Plan de Ordenamiento Territorial, el cual será analizado en el presente escrito, desde la participación de la sociedad civil de la comuna 21.

La articulación de la dimensión social al POT involucra, entonces, resignificar los esfuerzos del estado para garantizar resultados que converjan hacia el 
común de la sociedad. Por lo anterior, se requiere adelantar procesos abiertos de dialogo y discusión que empodere a la sociedad civil en la solución de sus propios problemas.

De acuerdo a los términos previstos en el artículo 4 de la Ley 388 de 1997 "las administraciones municipales, distritales y metropolitanas deberán fomentar la concertación entre los intereses sociales, económicos y urbanísticos, mediante la participación de los pobladores y sus organizaciones", para lo cual deberán adelantar espacios de concertación que garanticen la eficacia de las acciones emprendidas respecto de las necesidades de los diversos sectores de la población frente al ordenamiento territorial del Municipio.

En ese orden de ideas, Hernández (2010) afirma con relación a la construcción de los POT que "La característica esencial es que sean construidos colectivamente y a partir de un proceso de participación social que permita discutir ese orden deseado que se quiere en el territorio. Aquí está el punto neurálgico sobre la construcción del orden deseado", de esta manera la sociedad esta empoderada del conocimiento necesario para tomar decisiones acertadas sobre las estrategias y el presupuesto requerido, en procura de atender las dinámicas sociales evidenciadas.

La ciudad de Santiago de Cali, capital del Valle del Cauca, es considerada como uno de los centros industriales, económicos y sociales más importantes del País y la tercera en orden de importancia, ubicada en la región Sur del Valle del Cauca, entre la cordillera occidental y la cordillera central de los Andes; con una población en el 2016 de 2.394.870 habitantes, de acuerdo a las estimaciones del Departamento Administrativo Nacional de Estadística (2010).

Dicha ciudad posee una riqueza incalculable en flora y fauna, facilitando su desarrollo social. No obstante, situaciones sociales como inseguridad alimentaria, baja cobertura en servicios de salud, escases de agua potable, entre otras; hacen de la ciudad un escenario proclive a problemas sociales, que merecen ser abordados bajo las premisas arriba descritas; en aras de dar respuesta a las problemáticas de ciudad, que afrontan principalmente los sectores más vulnerables.

La investigación evidencia la falta de promoción de espacios de discusión del POT y por ende una baja participación ciudadana. De esta manera se genera un notorio distanciamiento entre los ejercicios derivados de la construcción del POT y la sociedad civil. 
La ciudad de Cali intenta superar las crisis económicas y sociales que afronta, pero aún falta voluntad política, que agencie la participación ciudadana entre sus habitantes, aunque los fenómenos migratorios, la violencia generada en las comunas deprimidas, la corrupción política, hayan convertido este territorio en un escenario violento para lograr la paz y la justicia social, que promueve el ordenamiento territorial, no obstante una luz en el camino sería evidenciar la búsqueda de alternativas investigativas y educativas que permitan que la estrategia del plan de ordenamiento territorial como lo establece en uno de sus apartes: "Logrando de Santiago de Cali un territorio líder, innovador, incluyente, que le apuesta a la población como centro de sus decisiones, priorizando el talento, la disciplina, la dedicación, el desarrollo de sus aspectos propios de biodiversidad multicultural y pluriétnicas de su población". Concejo Municipal, Plan de Ordenamiento Territorial Santiago de Cali (2014).

\section{MATERIALES Y MÉTODOS}

Para la consecución de los objetivos propuestos para esta primera fase, en función de la participación civil en la construcción tanto del POT, se desarrolló una investigación descriptiva, en aras de establecer la participación de la población en relación al ordenamiento territorial. Se empleó un cuestionario con 7 preguntas, en las cuales se indaga sobre la participación en la construcción del POT de la ciudad de Cali.

Se tomó como referencia la comuna 21, localizada al sur del área urbana, en la ribera del río cauca, dicha comuna está conformada por 8 barrios y 6 urbanizaciones. De acuerdo con proyecciones de la Alcaldía de Cali (2010), la población de la comuna es de 100.819 habitantes, así mismo el 80\% de las viviendas pertenece a estrato 1.

La aplicación del instrumento de recolección de la información fue aplicada en el primer semestre de 2016, analizando la participación y reconocimiento de la muestra poblacional frente al POT de Cali aprobado a finales de 2014 y con vigencia hasta diciembre de 2027.

La comuna fue seleccionada como zona de influencia de la investigación, en tanto que es considerada como una de las zonas más reprimidas de la ciudad de Cali, caracterizada por los altos niveles de violencia e inseguridad.

Dichos elementos fueron considerados claves, toda vez que la investigación tiene la intención de establecer el conocimiento y participación de la población más vulnerable en los POT. 
Con un nivel de confianza del 95\% y mediante un muestreo aleatorio, se tomó como referencia una muestra representativa de 400 personas, a las cuales se les aplicó el instrumento, que se presenta a continuación.

\section{RESULTADOS}

Tabla 1

GÉNERO DEL ENCUESTADO

\begin{tabular}{lcccc}
\hline & Frecuencia & Porcentaje & $\begin{array}{c}\text { Porcentaje } \\
\text { válido }\end{array}$ & $\begin{array}{c}\text { Porcentaje } \\
\text { acumulado }\end{array}$ \\
\hline Femenino & 168 & 42 & 42 & 42 \\
Masculino & 232 & 58 & 58 & 100 \\
\hline Total & 400 & 100 & 100 & \\
\hline
\end{tabular}

Fuente: Los autores.

Las encuestas se aplicaron a un total de 400 personas de las cuales 232 son hombres y 168 mujeres, en un porcentaje de $58 \%$ y $42 \%$, respectivamente. La paridad proporcional entre los encuestados da lugar a establecer que las respuestas no están sesgadas por género.

Tabla 2

NIVEL DE FORMACIÓN DE LOS ENCUESTADOS

\begin{tabular}{lcccc}
\hline & Frecuencia & Porcentaje & $\begin{array}{c}\text { Porcentaje } \\
\text { válido }\end{array}$ & $\begin{array}{c}\text { Porcentaje } \\
\text { acumulado }\end{array}$ \\
\hline Bachillerato & 204 & 51 & 30 & 30 \\
Técnico & 72 & 18 & 18 & 48 \\
Tecnológico & 68 & 17 & 17 & 65 \\
Universitario & 56 & 14 & 14 & 79 \\
Postgrado & 0 & 0 & 0 & 100 \\
\hline Total & 400 & 100 & 100 & \\
\hline
\end{tabular}

Fuente: Los autores. 
De acuerdo con la población encuestada, el 18\% manifiesta que su nivel de formación es técnico, el 17\% acredita formación en educación básica media y tecnológica, mientras que el $14 \%$ restante es de nivel universitario.

Destaca el hecho de que el 51\% de la muestra acredite bachillerato como máximo nivel de formación, hecho el cual está relacionado con factores socioeconómicos, siendo dicha comuna habitada por personas en condición de vulnerabilidad.

El tener un grupo tan diverso y con formación en los diferentes niveles académicos enriquece y a su vez valida los resultados arrojados por la presente investigación.

Tabla 3

¿CONOCE QUE ES UN PLAN DE ORDENAMIENTO

TERRITORIAL POT?

\begin{tabular}{lcccc}
\hline Frecuencia & Porcentaje & $\begin{array}{c}\text { Porcentaje } \\
\text { válido }\end{array}$ & $\begin{array}{c}\text { Porcentaje } \\
\text { acumulado }\end{array}$ \\
\hline $\mathrm{Si}$ & 104 & 26 & 26 & 26 \\
No & 296 & 74 & 74 & 100 \\
NS/NR & 0 & 0 & 0 & \\
\hline Total & 400 & 100 & 100 & \\
\hline
\end{tabular}

Fuente: Los autores.

A partir de los resultados se observa que el $74 \%$ de la muestra seleccionada desconoce que es el Plan de Ordenamiento Territorial, siendo un motivo de preocupación, toda vez que el marco normativo y regulatorio establece la necesidad de fomentar la concertación de los distintos intereses, mediante la participación de la sociedad civil, tal como lo establece la Ley 388 de 1997 en el artículo 4.

Sin embargo, los resultados muestran una tendencia que ratifica la necesidad de generar mayor divulgación en la población sobre las características del POT y su importancia dentro de la planificación y ordenamiento del desarrollo, dando lugar a procesos abiertos de carácter participativo que respondan a las necesidades de la población. 
Tabla 4

¿CONOCE EL POT DE LA CIUDAD DE CALI?

\begin{tabular}{lcccc}
\hline & Frecuencia & Porcentaje & $\begin{array}{c}\text { Porcentaje } \\
\text { válido }\end{array}$ & $\begin{array}{c}\text { Porcentaje } \\
\text { acumulado }\end{array}$ \\
\hline $\mathrm{Si}$ & 70 & 17,5 & 17,5 & 17,5 \\
$\mathrm{No}$ & 330 & 82,5 & 82,5 & 100 \\
$\mathrm{NS} / \mathrm{NR}$ & 0 & 0 & 0 & \\
\hline Total & 400 & 100 & 100 & \\
\hline
\end{tabular}

Fuente: Los autores.

Es preocupante identificar que el $82,5 \%$ de los encuestados no conocen el POT de la ciudad de Cali, el cual fue revisado y aprobado el contenido de largo plazo, mediante el Acuerdo $N^{\circ} 0373$ de 2014.

Lo anterior teniendo en cuenta la obligatoriedad de garantizar, de acuerdo con el artículo 24 de la Ley 388 de 1997; espacios de publicidad y divulgación del proyecto en sus diferentes fases (diagnóstico, aprobación, seguimiento y evaluación), garantizando que la sociedad civil conozca oportunamente sobre el contenido del mismo, motivando un proceso de construcción colectiva.

Se requiere entonces reevaluar los mecanismos de difusión que se desarrollaron en aras de alcanzar una mayor cobertura y efectividad.

Tabla 5

¿PARTICIPÓ EN LAS DISCUSIONES PARA LA ELABORACIÓN DEL POT?

\begin{tabular}{lcccc}
\hline & Frecuencia & Porcentaje & $\begin{array}{c}\text { Porcentaje } \\
\text { válido }\end{array}$ & $\begin{array}{c}\text { Porcentaje } \\
\text { acumulado }\end{array}$ \\
\hline $\mathrm{Si}$ & 0 & 0 & 0 & 0 \\
$\mathrm{No}$ & 400 & 100 & 100 & 100 \\
$\mathrm{NS} / \mathrm{NR}$ & 0 & 0 & 0 & \\
\hline Total & 400 & 100 & 100 & \\
\hline
\end{tabular}

Fuente: Los autores. 
Los resultados alcanzados denotan claramente que, de acuerdo a la población analizada, fue nula la participación en las instancias de concertación y consulta referidas en la Ley en comento.

Valga la pena resaltar que es deber del legislativo promover espacios de discusión y reflexión que alberguen a la población, principalmente aquella con mayores externalidades, para conocer sus percepciones y posibles sugerencias, las cuales han de ser consideradas y debatidas a la luz de criterios de factibilidad, entre otros, y que suponen un primer paso hacia la consolidación de una agenda pública incluyente que den respuesta a las necesidades sentidas por la población.

Es prioritario entonces promover una mayor conciencia en la sociedad civil para que a través de los diversos canales de participación ciudadana se ejerza el derecho a ser partícipe de la construcción de ciudad.

Tabla 6

¿CONOCE LAS IMPLICACIONES QUE TENDRÁ EL POT PARA SU COMUNA?

\begin{tabular}{lcccc}
\hline & Frecuencia & Porcentaje & $\begin{array}{c}\text { Porcentaje } \\
\text { válido }\end{array}$ & $\begin{array}{c}\text { Porcentaje } \\
\text { acumulado }\end{array}$ \\
\hline $\mathrm{Si}$ & 42 & 10,5 & 10,5 & 10,5 \\
$\mathrm{No}$ & 358 & 89,5 & 89,5 & 100 \\
$\mathrm{NS} / \mathrm{NR}$ & 0 & 0 & 0 & \\
\hline Total & 400 & 100 & 100 & \\
\hline
\end{tabular}

Fuente: Los autores.

Con ocasión a un desconocimiento generalizado sobre el POT de la ciudad de Cali, es notorio que el 89,5\% de los encuestados no tengan claridad sobre las externalidades positivas y negativas que tendrá dicho instrumento para la comuna donde habita.

Se deberá entonces establecer procesos de comunicación focalizados hacia las comunas, para que, desde las particularidades de ellas, en función a los atributos del instrumento de planeación, se genere empoderamiento en los líderes comunitarios para desarrollar control y veeduría ciudadana hacia los resultados alcanzados. 
Tabla 7

¿CONSIDERA USTED QUE EL POT AFECTA SU

CALIDAD DE VIDA?

\begin{tabular}{lcccc}
\hline & Frecuencia & Porcentaje & $\begin{array}{c}\text { Porcentaje } \\
\text { válido }\end{array}$ & $\begin{array}{c}\text { Porcentaje } \\
\text { acumulado }\end{array}$ \\
\hline $\mathrm{Si}$ & 50 & 12,5 & 12,5 & 12,5 \\
$\mathrm{No}$ & 90 & 22,5 & 22,5 & 35 \\
$\mathrm{NS} / \mathrm{NR}$ & 260 & 65 & 65 & 100 \\
\hline Total & 400 & 100 & 100 & \\
\hline
\end{tabular}

Fuente: Los autores.

Los resultados alcanzados demuestran que el $12,5 \%$ de la muestra seleccionada, considera que el POT les afecta la calidad de vida. Teniendo en su mayoría el desconocimiento de lo que puede afectar el POT en la calidad de vida, con 65\% de los encuestados.

Dichos resultados da lugar a concluir que existe un precario conocimiento sobre la génesis y función de la política de ordenamiento territorial, que guiara el desarrollo de la ciudad de Cali hasta el año 2027, toda vez que el mismo define aspectos trascendentales propios a la expansión físico territorial del municipio, así como las actividades productivas, culturales y de esparcimiento que garantizan el mejoramiento de la calidad de vida de sus habitantes, al acceder a los distintos beneficios que ofrece el desarrollo de la ciudad, velando por la debida protección y conversación del ecosistema, lo cual debe facilitar un desarrollo de beneficio común desde la sostenibilidad.

\section{ANÁLISIS DE RESULTADOS}

El Plan de Ordenamiento Territorial expedido por el Congreso de la Republica de Colombia mediante la Ley 388 de 1997, establece en el artículo 4 que el ordenamiento del territorio, es un instrumento de agenda pública, concertado de manera participativa con la sociedad civil, garantizando una serie de beneficios intergeneracionales que dé cuenta de las necesidades sentidas por parte de la población. 
Igualmente, la Ley en comento, y en aras de alcanzar lo mencionado en el párrafo anterior, el artículo 22 se establece el deber de crear espacios de publicidad y divulgación del proyecto para garantizar la participación de la sociedad civil en las distintas etapas.

Sin embargo, la investigación evidencio que el $64 \%$ de los encuestados no tiene conocimiento sobre el Plan de Ordenamiento Territorial, mientras que el $82,5 \%$ de los encuestados no conocen el POT de la ciudad de Cali.

De acuerdo con el Departamento Nacional de Planeación (1999), los Planes de Ordenamiento Territorial debían garantizar la incorporación y alcance de diversos elementos del desarrollo y la ocupación del espacio físico urbano en consonancia un desarrollo sostenible; sin embargo, la poca o casi nula incorporación de la sociedad civil en los temas de Ordenamiento Territorial ponen en condiciones críticas un accionar conjunto hacia el beneficio de la sostenibilidad. Dicho de otra manera, al no tenerse en cuenta a la sociedad civil, es posible que el ordenamiento territorial presente a futuro acciones contradictorias entre el estado y a quienes se les debe favorecer, la población civil.

Así pues dicho instrumento tiene el objetivo de "orientar la distribución actual y futura de las actividades en el espacio, buscando racionalizar y articular las políticas sectoriales para alcanzar una distribución más equilibrada del desarrollo y para regular la ocupación del suelo, protegiendo y aprovechando sosteniblemente los recursos naturales" Departamento Nacional de Planeación (2013). En esta misma línea, el acontecer a futuro de la sostenibilidad dentro del marco político y social igualmente se verá forzado a debates sociales que pugnarán por un lado por el beneficio social, pero por otro lado, por las políticas económicas de las cuales se verá alcanzado el presupuesto municipal.

Tal situación pone de manifiesto entonces una brecha entre los preceptos establecidos por el marco normativo y su aplicación, por cuanto la tendencia observada pone de manifiesto la necesidad de informar activamente a la población, empoderándolos hacia un proceso de carácter colectivo.

Igualmente se observa la resistencia de la sociedad civil hacia los procesos políticos y la creación de la agenda pública, lo cual trae como resultado una sociedad altamente desinformada.

Se requiere abrir nuevos espacios de discusión sobre el ordenamiento territorial, concientizando a la población sobre la importancia del mismo y sus impli- 
caciones desde la sostenibilidad, toda vez que el 100\% de la muestra asevera que no fue participe en las discusiones sobre el POT de Cali.

Con estos resultados el legislativo esta en mora de promover nuevas estrategias de comunicación sobre las afectaciones derivadas de las estrategias y programas propuestos, permitiendo que la sociedad civil pueda formular y generar nuevas estrategias de desarrollo y otras medidas de compensación o mitigación, que han de ser evaluadas a la luz de criterios de factibilidad, entre otros y que suponen un primer paso hacia la consolidación de una agenda pública incluyente que den respuesta a las necesidades sentidas por la población.

El hecho de que el 89,5\% de los encuestados no tengan claridad sobre los efectos positivos y negativos que tendrá dicho instrumento para la comuna donde habita y de que el $65 \%$ de los encuestados no tenga claridad sobre si el POT pueda posiblemente afectar la calidad de vida, deja en entredicho la intencionalidad de un verdadero ejercicio de política pública, en el que la población mediante los mecanismos previstos por la Constitución y demás Leyes, direccione su propio desarrollo y regule las diferentes actividades comerciales, productivas, culturales, entre otras que convergen en el espacio territorial, en donde es de vital importancia fomentar los espacios de rendición de cuentas y veeduría ciudadana, como control a lo dispuesto desde los Planes de Ordenamiento, Planes de Desarrollo, entre otros.

Difícilmente se ha de alcanzar un escenario de sostenibilidad en donde la dimensión social se encuentra altamente desarticulada del escenario político, como es evidente mediante esta investigación

\section{CONCLUSIONES}

El Plan de Ordenamiento Territorial, de acuerdo a sus características, establece un conjunto de acciones de orden político administrativo atenientes al desarrollo del territorio, el aprovechamiento del espacio a partir de estrategias de orden económico, social y ambiental, las cuales por su impacto en los habitantes deben ser necesariamente divulgadas a la población, garantizando su activa participación en las discusiones del mismo para lograr asíla legitimización de sus estrategias por parte de la sociedad civil.

La brecha organizacional surgida en la imposición de espacios públicos a manera de desarrollo sostenible, han sido poco consultadas con la sociedad civil, que, de acuerdo con la Ley Orgánica de Ordenamiento Territorial, la manera de 
darle viabilidad a los procesos de desarrollo que a futuro favorezcan de manera equitativa solo pueden ser viables en la medida que surjan caminos concertados entre la sociedad civil y el estado.

Es menester de acuerdo a la normatividad nacional, garantizar la activa participación de la comunidad, disponiendo todos aquellos canales y recursos que sean necesarios para lograr establecer acuerdos de voluntades que propugnen mediante acuerdos gana un verdadero compromiso de la sociedad civil. Con lo anterior se logrará la atención de la dimensión social, la cual, articulada a los instrumentos económicos, el reconocimiento y la protección de los bienes y servicios ambientales, dará como resultado alcanzar un escenario de sostenibilidad enmarcado en valores de equidad, justicia y desarrollo social.

La investigación adelantada en la comuna 21, evidencia la falta de promoción de espacios, por un lado y de participación ciudadana por el otro, toda vez que los resultados reflejan un notorio distanciamiento entre los ejercicios derivados de la construcción del POT y el conocimiento como resultado de la participación por parte de la sociedad civil. Así pues, difícilmente se ha de alcanzar un escenario de sostenibilidad en donde la dimensión social se encuentra altamente desarticulada de las actuaciones del estado en cabeza de sus poderes, principalmente el legislativo y ejecutivo.

En las actuales circunstancias que vive el territorio colombiano, a puertas del posconflicto generados en los diálogos de paz, debe trazarse rutas para los nuevos retos sociales, que deben hilar menudo hacia la apertura de espacios de participación que involucre el tejido social con propiedad. A esos mecanismos acertados de construcción ciudadana, que promulgó la Carta Magna de 1991. En procura de construir una patria equitativa, incluyente, armónica y solidaria, pero sobre todo promoviendo un desarrollo urbano sostenible, donde la responsabilidad social, sea el ejercicio constante, para ello es necesario que las instituciones educativas de educación superior apuesten por investigaciones sociales reales, para insertar la academia en la construcción ciudadana.

La ciudad de Cali intenta superar las crisis económicas y sociales que afronta, pero aún falta la voluntad política, que agencie la participación ciudadana entre sus habitantes, aunque los fenómenos migratorios, la violencia generada en las comunas deprimidas, la corrupción política, hayan convertido este territorio en un escenario violento para lograr la paz y la justicia social, que promueve el ordenamiento territorial, no obstante una luz en el camino sería evidenciar la 
búsqueda de alternativas investigativas y educativas que permitan que la estrategia del plan de ordenamiento territorial como lo establece en uno de sus apartes: "Logrando de Santiago de Cali un territorio líder, innovador, incluyente, que le apuesta a la población como centro de sus decisiones, priorizando el talento, la disciplina, la dedicación, el desarrollo de sus aspectos propios de biodiversidad multicultural y pluriétnicas de su población". Concejo Municipal, Plan de Ordenamiento Territorial Santiago de Cali (2014).

\section{REFERENCIAS BIBLIOGRÁFICAS}

Alcaldía de Santiago de Cali. (2010). Cali en cifras 2010. Recuperado de http://planeacion.cali.gov.co/Publicaciones/Cali_en_Cifras/Caliencifras2010.pdf

Concejo Municipal Santiago de Cali. (2014). Plan de Ordenamiento Territorial. Recuperado de: http://www.cali.gov.co/publicaciones/106497/pot_2014_idesc/

Congreso de Colombia. (18 de julio de 1997). Ley de Ordenamiento Territorial. (Ley 388 de 1997). D.O: 43.091 .

Departamento Administrativo de Planeación Municipal (1999). Plan de Ordenamiento Territorial. Recuperadodehttp://www.cali.gov.co/publico2/pot/documentos/documentosoporte.pdf

Departamento Administrativo de Planeación Municipal. (2013). Lineamientos para el proceso de Ordenamiento Territorial Departamental contenidos básicos. Departamento Nacional de Planeación. Recuperadode http://www.usbcali.edu.co/sites/default/files/lineamientosdelacot.pdf.

Departamento Administrativo Nacional de Estadística (2010). Estudios postcensales: proyecciones nacionales y departamentales de población 2005-2020, Bogotá. Recuperado de: $<$ https://www.dane.gov.co/files/investigaciones/poblacion/proyepobla06_20/7Proyecciones_p oblacion.pdf $>$

Devia, S.P. (2008). La descentralización en Colombia: Un reto permanente. Revista de Información básica, 1 (2). Bogotá.

Hernández, Y.T. (2010). El ordenamiento territorial y su construcción social en Colombia: ¿un instrumento para el desarrollo sustentable? Cuadernos de Geografía (19).

República de Colombia. (1991). Constitución Política de Colombia, Bogotá (Colombia).

Rodríguez, C. y Reyes, S. (2008). Propuesta Metodológica para la elaboración de un Plan de Ordenamiento Territorial Sustentable. Proyección 4. 1, (4), Chile. Recuperado de http://bdigital.uncu.edu.ar/objetos_digitales/3240/seegerproyeccion4.pdf 